\title{
Medical Algorithm: Diagnosis and Managemen of Humoral Immunodeficiencies
}

\author{
Anna Šedivá ${ }^{1}$, Tomas Milota ${ }^{2}$, Jiri Litzman ${ }^{3}$, Isabella Quinti $^{4}$, Isabelle Meyts ${ }^{5}$, Siobhan \\ Burns $^{6}$, and Stephen Jolles ${ }^{7}$ \\ ${ }^{1}$ University Hospital Motol \\ ${ }^{2}$ Univerzita Karlova v Praze 2 lekarska fakulta \\ ${ }^{3}$ St Anne's University Hospital \\ ${ }^{4}$ Universita degli Studi di Roma La Sapienza Dipartimento di Medicina Clinica \\ ${ }^{5} \mathrm{KU}$ Leuven University Hospitals Leuven \\ ${ }^{6}$ University College London \\ ${ }^{7}$ University Hospital of Wales
}

February 1, 2021

\section{Medical Algorithm: Diagnosis and Managemen of Humoral Immunodeficiencies}

Anna Šedivá ${ }^{1}$, Tomáš Milota ${ }^{1}$, Jiří Litzman ${ }^{2}$, Isabella Quinti ${ }^{3}$, ESID Clinical Working Party, EAACI Primary Immunodeficiency Working Group, Stephen Jolles ${ }^{4}$

1. Department of Immunology, 2nd Faculty of Medicine Charles University, V Uvalu, University Hospital in Motol, Prague, Czech Republic

2. Faculty of Medicine, Masaryk University Brno, Czech Republic; Department of Clinical Immunology and Allergology, St Anne's University Hospital, Brno, Czech Republic

3. Department of Molecular Medicine, Sapienza University of Rome, Rome, Italy

4. Immunodeficiency Centre for Wales, University Hospital of Wales, Cardiff, UK

For ESID Clinical Working Party:

Isabelle Meyts, Department of Pediatrics, Leuven University Hospitals, Leuven, Belgium

Siobhan Burns, Institute of Immunity and Transplantation, University College London, London, United Kingdom; Department of Immunology, Royal Free London NHS Foundation Trust, London, United Kingdom

Author's contributions:

AS prepared the concept and wrote the manuscript, TM edited Figures and wrote the manuscript, JL designed Figures and revised manuscript, IQ designed Figures and revised the manuscript, IM revised manuscript, SB revised manuscript, ST edited Figures and wrote the manuscript.

\section{Background}

Primary antibody deficiencies (PAD) constitute the majority of all primary immunodeficiency diseases (PID) also termed inborn errors of immunity (IEI). This category (PAD) represents around $52 \%$ of all IEI and the proportion overall is still greater given that antibody deficiency is a component of other groups including combined immunodeficiencies (CID and SCID), autoinflammatory disorders, diseases of immune dysregulation and other well defined PIDs $(1,2)$. Secondary antibody deficiencies (SAD) represent a larger and expanding number of individuals resulting from the use of a wide range of immunosuppressive therapies, in particular 
those targeting B cells, and may also result from renal or gastrointestinal immunoglobulin losses, infections (HIV, malaria) and globally malnutrition (3).

The manifestations of PADs are protean and encompass a range of infectious and non-infectious complications, including autoimmune, lymphoproliferative, granulomatous and allergy manifestations due to an immunedysregulation, and increased risk of malignancy in some forms of PADs $(4,5)$.

\section{Diagnosis}

The diagnosis is based on a combination of clinical features together with laboratory findings, potentially a positive family history and increasingly, genetic testing. The clinical presentation for antibody deficiency is often characterised by recurrent sinopulmonary infections which often take longer to resolve requiring prolonged or intravenous antibiotic courses. In addition to the infections presentations complications may include autoimmune cytopenias, lymphoproliferation, non-infectious inflammation and allergy linked to underlying immune dysregulation. Primary antibody deficiency disorders are also linked to an increase in malignancy in particular lymphoma and gastric cancers $(4,6)$.

Initial laboratory assessment is to determine the levels of immunoglobulins (IgG, $\operatorname{IgA}$ and $\operatorname{IgM}$ ) alongside functional information on vaccine responses and lymphocyte enumeration in particular B cells (Figure 1). The commonest clinically relevant primary antibody deficiency of adults is Common variable immunodeficiency (CVID) and if diagnostic criteria (7) are fulfilled the decision to commence immunoglobulin replacement therapy (IgRT) is usually straightforward. However careful assessment and monitoring is required for both IgRT and the non-infectious complications. Genetic testing is now of key importance, to where possible define a molecular diagnosis allowing optimization and personalisation of therapy where pathway-specific approaches are indicated. An example of this is the use of abatacept in CTLA4 deficiency. There have also been major advances in the screening of newborns using TREC and KREC assays and in adults using calculated globulin $(8,9)$

\section{Management}

In patients with PADs, IgRT forms the mainstay of therapy for many patients $(10,11)$ (Figure 2). The decision to commence IgRT is clearer in well-defined PADs with a significant infection burden and supportive laboratory findings - hypogammaglobulinaemia with impaired response to vaccination. The decision is more complex if these findings are not all present such as hypogammaglobulinaemia with preserved vaccine responses or specific antibody deficiency.

IgRT may be administered either intravenously, subcutaneously or as a facilitated subcutaneous infusion using hyaluronidase and the decisions regarding route and frequency of administration and site of administration (home or hospital) are individual and are made jointly with the patient and medical team (12). In general, IgRT is commenced based on weight at $0.4-0.6 \mathrm{~g} / \mathrm{kg} / \mathrm{month}$ of IgG and is the adjusted on clinical (such as infection burden, bronchiectasis, type of PAD and other complications) and laboratory grounds including trough IgG level aiming for $>7 \mathrm{glL}$ (though this may vary between centres and countries) $(13,14)$. In a number of circumstances antibiotic prophylaxis may be used, such as potentially milder immunodeficiencies with a less severe infection burden (IgG subclass deficiency or combined IgA deficiency with IgG subclass deficiency), or in addition to IgRT in patients with a persistently increased infection burden despite optimally individualised IgG trough levels and in those with existing end-organ damage such as bronchiectasis or chronic sinusitis or in those colonised with Pseudomonas aeruginosa or Stenotrophomonas maltophilia . Antibiotic regimens will vary according to the setting, organism and sensitivities, but in PAD patients with frequent respiratory tract infections on IgRT who may also have bronchiectasis azithromycin at $250-500 \mathrm{mg}$ three days/wk has been shown to decrease infective exacerbations. In patients with concomitant T cell or neutrophil impairment (CID, SCID, HIGM), septrin (cotrimoxazole) may be considered $(10,15)$. PAD patients require regular follow up both to monitor therapy and for early detection and management of complications $(4,5,16,17)$. Laboratory and radiological monitoring are indvidualised but baseline testing includes full blood count, renal and liver function and CRP 2-4 times per year (with LDH, $\beta 2$ microglobulin as indicated), lymphocyte subsets 1-2 times per year, abdomen ultrasonography, neck ultrasonography if 
lymphadenopathy is investigated, chest X-ray and lung function tests once a year, chest CT every 3-5 years or sooner if indicated and screening endoscopies every 1-2 years in some centres (18).

Diagnosis and management are in PAD improving with better immunologic and molecular characterisation, more options for IgRT, personalised pathway-specific therapies for some PADs and greater knowledge of the role of HSCT and gene therapy/editing for the future.

\section{References}

1. Seidel MG, Kindle G, Gathmann B, Quinti I, Buckland M, van Montfrans J, et al. The European Society for Immunodeficiencies (ESID) Registry Working Definitions for the Clinical Diagnosis of Inborn Errors of Immunity. J Allergy Clin Immunol Pract. 2019;

2. Bousfiha A, Jeddane L, Picard C, Al-Herz W, Ailal F, Chatila T, et al. Human Inborn Errors of Immunity: 2019 Update of the IUIS Phenotypical Classification. J Clin Immunol. 2020;

3. Patel SY, Carbone J, Jolles S. The expanding field of secondary antibody deficiency: Causes, diagnosis, and management. Frontiers in Immunology. 2019.

4. Kralickova P, Milota T, Litzman J, Malkusova I, Jilek D, Petanova J, et al. CVID-associated tumors: Czech nationwide study focused on epidemiology, immunology, and genetic background in a cohort of patients with CVID. Front Immunol. 2019;

5. Ho HE, Cunningham-Rundles C. Non-infectious Complications of Common Variable Immunodeficiency: Updated Clinical Spectrum, Sequelae, and Insights to Pathogenesis. Front Immunol. 2020;

6. Hauck F, Voss R, Urban C, Seidel MG. Intrinsic and extrinsic causes of malignancies in patients with primary immunodeficiency disorders. J Allergy Clin Immunol. 2018;

7. Bonilla FA, Barlan I, Chapel H, Costa-Carvalho BT, Cunningham-Rundles C, de la Morena MT, et al. International Consensus Document (ICON): Common Variable Immunodeficiency Disorders. Journal of Allergy and Clinical Immunology: In Practice. 2016.

8. Korsunskiy I, Blyuss O, Gordukova M, Davydova N, Zaikin A, Zinovieva N, et al. Expanding TREC and KREC Utility in Primary Immunodeficiency Diseases Diagnosis. Front Immunol. 2020;

9. Jolles S, Borrell R, Zouwail S, Heaps A, Sharp H, Moody M, et al. Calculated globulin (CG) as a screening test for antibody deficiency. Clin Exp Immunol. 2014;

10. Hanitsch L, Baumann U, Boztug K, Burkhard-Meier U, Fasshauer M, Habermehl P, et al. Treatment and management of primary antibody deficiency: German interdisciplinary evidence-based consensus guideline. Eur J Immunol. 2020;

11. Jolles S, Chapel H, Litzman J. When to initiate immunoglobulin replacement therapy (IGRT) in antibody deficiency: a practical approach. Clinical and Experimental Immunology. 2017.

12. Jolles S, Orange JS, Gardulf A, Stein MR, Shapiro R, Borte M, et al. Current treatment options with immunoglobulin $\mathrm{G}$ for the individualization of care in patients with primary immunodeficiency disease. Clinical and Experimental Immunology. 2015.

13. Orange JS, Grossman WJ, Navickis RJ, Wilkes MM. Impact of trough IgG on pneumonia incidence in primary immunodeficiency: A meta-analysis of clinical studies. Clin Immunol. 2010;

14. Orange JS, Belohradsky BH, Berger M, Borte M, Hagan J, Jolles S, et al. Evaluation of correlation between dose and clinical outcomes in subcutaneous immunoglobulin replacement therapy. Clin Exp Immunol. 2012 ;

15. Milito C, Pulvirenti F, Cinetto F, Lougaris V, Soresina A, Pecoraro A, et al. Double-blind, placebocontrolled, randomized trial on low-dose azithromycin prophylaxis in patients with primary antibody deficiencies. J Allergy Clin Immunol. 2019; 
16. U. B, J.M. R, P. S-P, S. J. The lung in primary immunodeficiencies: New concepts in infection and inflammation. Front Immunol. 2018;

17. van de Ven AAJM, Alfaro TM, Robinson A, Baumann U, Bergeron A, Burns SO, et al. Managing Granulomatous-Lymphocytic Interstitial Lung Disease in Common Variable Immunodeficiency Disorders: e-GLILDnet International Clinicians Survey. Front Immunol. 2020;

18. Jolles S, Sánchez-Ramón S, Quinti I, Soler-Palacín P, Agostini C, Florkin B, et al. Screening protocols to monitor respiratory status in primary immunodeficiency disease: findings from a European survey and subclinical infection working group. Clin Exp Immunol. 2017;

Figure 1: Diagnostic algorithm for hypogammaglobulinemia (PID- Primary antibody deficiency, CVIDCommon variable immunodeficiency, THI- Transient hypogammaglobulinemia of infancy, SPAD- Specific antibody deficiency, XL- X-linked, AD- Autosomal dominant, AR- Autosomal recessive, GOF- Gain-offunction)

Figure 2: Therapeutic algorithm for hypogammaglobulinemia (ATB- Antibiotics, IgRT- Immunoglobulin replacement therapy, AI- Aitoimmune, GLILD- Granulomatous-lymphocytic interstitial lung disease, IBDInflammatory bowel disease, LN- Lymph node)

\section{Hosted file}

Hypogamma_Diagnostic and Therapeutic algorithm_ALLERGY Jan 2021 final.pptx available at https://authorea.com/users/321819/articles/506841-medical-algorithm-diagnosis-andmanagemen-of-humoral-immunodeficiencies 